\title{
Facing the unique challenges of dyskinesias in Parkinson's disease
}

\section{Houman Homayoun* \& Christopher G Goetz}

Rush University Medical Center, Chicago, IL 60672, USA

*Author for correspondence: Suite 755, 1725 W. Harrison Street, Chicago, IL 60612, USA = Tel.: +1 3125634772

= Fax: +1 3125632024 m houman_homayoun@rush.edu

Dyskinesia is among the most challenging complications of levodopa and dopaminergic drug therapy in advanced Parkinson's disease. This symptom has a negative impact on the quality of life of patients with Parkinson's disease and is hard to manage. Current advances in our understanding of the diverse phenomenology and complicated pathophysiology of dyskinesia have led to a number of novel strategies aimed at better control of this complication. Further insight has been gained from focusing on the characteristics of the rating scale used for assessment of dyskinesia and from the inherent susceptibility of dyskinesia to placebo effect. Here, we will briefly review the phenomenology, pathophysiology and the treatment of dyskinesia in Parkinson's disease.

\section{Medscape Medscape: Continuing Medical Education Online}

This activity has been planned and implemented in accordance with the Essential Areas and policies of the Accreditation Council for Continuing Medical Education through the joint sponsorship of Medscape, LLC and Future Medicine Ltd. Medscape, LLC is accredited by the ACCME to provide continuing medical education for physicians.

Medscape, LLC designates this Journal-based CME activity for a maximum of 1 AMA PRA Category 1 Credit(s) ${ }^{\mathrm{TM}}$. Physicians should claim only the credit commensurate with the extent of their participation in the activity.

All other clinicians completing this activity will be issued a certificate of participation. To participate in this journal CME activity: (1) review the learning objectives and author disclosures; (2) study the education content; (3) take the post-test with a $70 \%$ minimum passing score and complete the evaluation at www.medscape.org/journal/fnl; (4) view/print certificate.

\section{Release date: 8 March 2012; Expiration date: 8 March 2013}

\section{Learning objectives}

Upon completion of this activity, participants should be able to:

- Describe the clinical features of dyskinesias in patients with advanced Parkinson disease

- Describe the risk factors for dyskinesias in Parkinson disease

- Describe the treatment of dyskinesias in patients with advanced Parkinson disease

\section{Financial \& competing interests disclosure}

Editor: Elisa Manzotti, Publisher, Future Science Group. Disclosure: Elisa Manzotti has disclosed no relevant financial relationships.

Authors \& credentials: Houman Homayoun, Rush University Medical Center, Chicago, IL 60612, USA. Disclosure: Houman Homayoun is employed by the Rush University Medical Center, a Parkinson's Disease Foundation Parkinson's Research Center. Christopher G Goetz, Rush University Medical Center, Chicago, IL 60612, USA. Disclosure: Christopher G Goetz receives consulting and advisory board membership with honoraria from Addex Pharma SA, Asubio, Biovail Technologies, Boston Scientific, Cleveland Medical Devices, Decision Resources, Dixon Group, ICON Clinical Research, Impax Pharmaceuticals, Ingenix (i3 Research), Intec Pharmaceuticals, Kenes International, Motac Neurosciences, Oxford Biomedica and United Bioscience Corporation (UCB). He receives funding from the NIH and the Michael J Fox Foundation. He directs the Rush Parkinson's Disease Research Center that receives support from the Parkinson's Disease Foundation. He directs the translation 
program for the MDS-UPDRS and UDysRS and receives funds from the MDS for this effort. He receives honoraria from the Movement Disorder Society, the American Academy of Neurology, the University of Pennsylvania, Washington University and the University of Michigan. He receives royalties from Oxford University Press, Elsevier Publishers, Wolters Kluwer Health, Lippincott, Wilkins and Williams. He is employed by the Rush University Medical Center, a Parkinson's Disease Foundation Parkinson's Research Center.

No writing assistance was utilized in the production of this manuscript.

CME author: Laurie Barclay, Freelance writer and reviewer, Medscape, LLC. Disclosure: L Barclay has disclosed no relevant financial relationships.

\section{Background}

Dyskinesias (from 'dys', difficult and 'kinesis', movement) are abnormal involuntary movements of various forms. In the setting of Parkinson's disease (PD), dyskinesia is a highly problematic complication that results from the interplay between the progressive course of the disease and dopaminergic drug therapy. These dyskinesias, often referred to as levodopainduced dyskinesias, can appear with levodopa treatment, but also with dopaminergic agents and with the introduction of monoamine oxidase (MAO) - or catechol-O-methyl transferase (COMT)-inhibitors in patients already treated with levodopa. The emergence of dyskinesia, along with other motor complications, such as wearing off and fluctuations, have a negative impact on the quality of life of patients with PD and may limit their ability to receive an optimal level of dopaminergic therapy. Dyskinesias are also associated with a significant increase in healthcare cost [1]. Current available treatments for dyskinesia, including both pharmacological and surgical methods, remain limited in scope, and there is a clear need to develop new therapeutic strategies to address this clinical problem.

To achieve this goal, several issues need to be addressed. First, dyskinesias differ in their clinical phenomenology including the type of abnormal movements as well as their temporal relationship to levodopa doses. A consideration of distinct types of dyskinesia is essential in current management and future drug development efforts. Second, the pathophysiology and pharmacology of dyskinesia is complex, and despite many advances in this field, they remain poorly understood. Recent studies have emphasized a number of candidate mechanisms underlying the development and expression of dyskinesias, leading to a number of strategies that are in various stages of testing. Finally, the dynamic and diverse nature of dyskinesias poses a challenge for their assessment in controlled clinical trials. For example, it has proved difficult to define target outcomes for objective assessment of dyskinesia although a number of rating scales have been developed and used with varying success. Moreover, placebo response can inherently affect the expression of dyskinesia and confound its assessment.

This review will focus on phenomenology, pathophysiology/neuropharmacology, and treatment issues related to medication-induced dyskinesia in PD. While it is recognized that the expression of dyskinesias in PD is not exclusive to levodopa and may also occur with other dopaminergic drugs, we will mainly focus on levodopa-induced dyskinesia as the most commonly encountered form in a clinical setting. From the onset, it is important to clarify that there are some forms of dyskinesia that are part of PD itself and not related to medication. A painful foot dystonia or calf cramping can occur in untreated PD and is a hallmark of the early disease. This same type of movement re-emerges later in the disease as part of a waning medication response to dopaminergic drugs, and in this instance, it is considered under the category of medication-related dyskinesia, although the pathophysiology of such movements likely relates to the same foundations as PD itself.

\section{Phenomenology}

Dyskinesia can be described based on the type of abnormal movements, anatomical areas involved and the temporal pattern of movements in relation to dopaminergic drug administration. The range of abnormal movements presenting as dyskinesia are broad and variable. Most commonly, dyskinesia presents in the form of choreic or dystonic movements or a combination of the two. Choreic movements involve typically fast, irregular, jerking movements of the face, neck and extremities, although the trunk can also become involved. Dystonic movements manifest most commonly as distorted posturing, but can also include mobile dystonia, craniocervical dystonia and blepharospasm [2]. Less commonly, dyskinesia may be seen in the form of stereotypies, myoclonus and respiratory or ocular movements [2-5]. Whereas occasionally authors have also included akathisia, restlessness and a worsening of baseline tremor in the spectrum 
of dyskinesia, these movement disorders are not considered dyskinesias in this discussion [6]. In assessing the severity of dyskinesia, it is important to note that motor tasks, mental calculation and emotional distress can all aggravate the severity of dyskinesia, requiring use of activation tasks, such as speaking aloud or concomitant movement, in order to objectively assess the severity and impact of dyskinesia in activities of daily living.

All body parts may be affected by dyskinetic movements although different subtypes of dyskinesia may have a predilection for certain body parts (see below). In general, involvement of the extremities, trunk and lingual-facial-buccal muscles are common with choreic dyskinesias but thoracic and abdominal muscles may occasionally be affected. Dystonic dyskinesia often affects the foot, but can also involve the neck and trunk. Cases of ocular dyskinesia have been reported with either large amplitude oscillations of gaze or conjugate upward and lateral gaze deviations [4]. Respiratory dyskinesia is another rare form of dyskinesia and consists of symptomatic dyspnea related to irregular tachypnea alternating with brief episodic apnea experienced after a dose of levodopa [5,7]. An unusual form of dyskinesia involves a bizarre stereotyped 'silly walk' gait that consists of stepping with kicks, high knee elevations, and ballistic stamping of one of the legs, and may be misinterpreted as a psychogenic gait [8]. Punding is a rare form of levodopa-induced motor stereotypy characterized by intense fascination with repetitive handling, examining, sorting and arranging of objects [9], although its classification as a form of dyskinesia remains questionable.

Based on their temporal patterns, dyskinesias can be divided into three main phenotypes: peak-dose dyskinesia, diphasic dyskinesia, and end of dose/OFF-state dystonia $[2,3,10,11]$. Peakdose dyskinesia typically coincides with the high dopaminergic drug effect during the peak of 'ON state' and primarily consists of choreic movements of the head, neck and upper extremities. Other abnormal movements including mobile dystonia can also be seen in combination with chorea and are usually painless. Peak-dose dyskinesia is believed to be related to the plateau plasma levels of levodopa in most instances.

By contrast, diphasic dyskinesia, also known as 'onset and end-of-dose dyskinesia', occurs at the beginning and end of a levodopa dose, separated by a dyskinesia-free interval. This type of dyskinesia is caused by fluctuations in the dopaminergic effects that are associated with rising and falling plasma levodopa levels. Thus, in diphasic dyskinesia the sequence of events following a dose of levodopa consists of initial emergence of dyskinesia usually when the patient's parkinsonism is still evident, then improvement of parkinsonism without prominent dyskinesia, and finally re-emergence of dyskinesia at the end of the dose as parkinsonism re-emerges. This dyskinesia-improvement-dyskinesia pattern is distinct from peak-dose dyskinesia in which the sequence is parkinsonism without dyskinesia, improvement in parkinsonism but the simultaneous appearance of dyskinesia, and then re-emergence of parkinsonism with reduction in dyskinesia. Diphasic dyskinesias primarily affect the trunk and lower extremities, often have a ballistic or dystonic appearance, and can be painful and disabling in terms of gait and balance. A common pattern involves stereotyped, rhythmic repetitive movements of the lower limbs with rocking of the trunk [2]. At times, determination of temporal relationship of dyskinesia to levodopa dosing may be difficult and can lead to the misdiagnosis of diphasic dyskinesia as peak-dose dyskinesia. This problem is more prominent in the afternoon where peak-dose dyskinesia related to accumulation of levodopa over the course of the day may be confused with diphasic dyskinesia related to poor postprandial absorption of levodopa [12].

End of dose/OFF-state dystonia usually involves the lower extremities and is believed to be related to low dopaminergic effect. There is a tendency to involve the limb most affected by parkinsonism first, presumably because of more severe dopaminergic denervation. In some patients, dystonic foot cramps are an early manifestation of untreated PD itself, and, with dopaminergic treatment, they resolve. When they emerge in the middle phases of PD in association with motor fluctuations, it is considered as a dyskinesia. This type of dyskinesia is correlated with akinesia in the OFF state and may involve painful inversion of the foot or extension of the toes. This type of dystonic foot may be the earliest manifestation of levodopa-induced dyskinesia and is related to early loss of projections from the ventrolateral substantia nigra to the somatotopic representation area of the foot in the dorsolateral subregion of the striatum [13]. While dystonic posturing is usually seen as a component of OFF-state dyskinesia, dystonic movement of limbs or craniocervical dystonia can occur as part of peak-dose dyskinesia [2]. The appearance of dystonia as the main manifestation of peak-dose dyskinesia, however, 
especially if early in the course of the disease and involving oromandibular muscles, is suggestive of multiple system atrophy [14]. Different phenomenologies of dyskinesias can occur in the same patient or in response to different doses of levodopa or other dopaminergic drugs.

\section{Epidemiology \& impact on quality of life}

Most studies have focused on levodopa exposure, and in these surveys, the prevalence of levodopa-related dyskinesia ranges from 30 to $80 \%$ in different studies [6]. This variability is likely attributable to different assessment methods and patient populations. The main risk factors associated with higher risk for development of dyskinesia include higher total dose and longer duration of levodopa therapy, higher severity of disease at the start of levodopa therapy, younger age at $\mathrm{PD}$ onset and female gender $[12,15]$. In a large community-based study, the emergence of dyskinesia correlated with duration of levodopa treatment such that the prevalence of dyskinesia increased from $11 \%$ in those with less than 5 years of levodopa therapy to $89 \%$ in those treated for 10 years or longer [16]. Dose of levodopa is a risk factor for the speed of development of dyskinesia as demonstrated in the prospective ELLDOPA study in which higher dose of levodopa was associated with a higher incidence of early onset dyskinesia at 6 months [17]. The relative dose of levodopa to body weight may also be a key factor and patients who receive a higher dose of levodopa per kilogram body weight, sometimes as a result of losing weight, are at higher risk for developing dyskinesia [15]. When dopamine receptor agonists are combined with levodopa, the cumulative dopaminergic dose is correlated with risk of dyskinesia, whereas in isolation these agonists cause dyskinesia much less frequently [18].

The severity of PD at the onset of levodopa therapy is another risk factor such that the average time between starting levodopa and onset of dyskinesia diminishes from 66 to 24 months in patients in stages I and III of PD, respectively [19]. It has also been shown that starting levodopa in very advanced PD patients naive to dopaminergic medications can lead to rapid onset dyskinesia, highlighting the role of dopaminergic denervation in this process [15]. Finally, younger age at PD onset is associated with higher incidence of dyskinesia [20]. It has been suggested that this factor may be mostly accounted for when correcting for levodopa dose and body weight [21]. It is also notable that dyskinesia occurs much more commonly in patients with
PD compared with atypical parkinsonism. This observation suggests that drug-related dyskinesias in PD likely involve postsynaptic dopamine receptors that are relatively preserved in PD but disrupted in atypical parkinsonism [12]. Studies on the quality of life of patients with PD indicate the negative impact of dyskinesia when they are moderate or severe in intensity. This impact is limited at early stages of PD where dyskinesias tend to be mild and nondisabling, but become prominent in the advanced stages of the disease, especially in the presence of painful and pervasive dyskinesias and as a result of subsequent limitations on the maximum tolerated dose of levodopa [1]. One study indicated that dyskinesia negatively affected quality of life in certain domains, including activities of daily living, communication, emotional wellbeing and bodily discomfort [22].

\section{Pathophysiology \& neuropharmacology}

Both clinical studies as well as experimental models, most notably lesioning studies using 1-methyl-4-phenyl-1,2,3,6-tetrahydropyridine (MPTP) in primates and 6-hydroxydopamine (6-OHDA) in rats, have helped advance our understanding of the pathophysiology of dyskinesia. It is now recognized that mechanisms underlying the initial development of dyskinesia after repeated doses of levodopa, a process also known as priming, are distinct from those that govern the expression of established dyskinesia that occurs in response to an acute challenge dose of levodopa.

The degeneration of the nigrostriatal dopaminergic pathway is generally considered a requisite for the development of dyskinesia. Normal subjects and non-PD patients without nigrostriatal degeneration (e.g., patients with dopa-responsive dystonia) rarely develop dyskinesias in response to dopaminergic drugs. Also as indicated above, the rate of development and severity of dyskinesia to a given dose of a dopaminergic drug correlates with the degree of dopaminergic denervation [19,23]. In MPTP-induced parkinsonian monkeys, dyskinesia develops only after $95 \%$ of dopamine neurons are depleted [24]. Exposure to levodopa, as an exogenous source of dopamine, or dopamine agonists, as agents that directly stimulate D2 dopamine receptors, is another important requisite for development of most forms of dyskinesia. The propensity for dyskinesia is much higher with levodopa compared with dopamine agonists. Unique pharmacokinetic and pharmacodynamic aspects of levodopa therapy are critical for this process. 
The pharmacokinetic hallmarks of levodopa are a short, 90 min elimination half-life and an erratic intestinal absorption pattern, leading to peaks and troughs in levodopa plasma level [25]. These fluctuations lead to a pulsatile and nonphysiological mode of dopaminergic stimulation within the striatum, a phenomenon that is augmented as the disease progresses. This feature of levodopa likely explains its much higher proclivity to induce dyskinesia in comparison to the dopamine agonists that have very long half-lives, with the exception of apomorphine. At the start of levodopa therapy, the relationship between plasma levodopa level and central dopaminergic effects remains nonlinear as compensatory mechanisms buffer the fluctuating plasma levels of levodopa and allow controlled and relatively physiologic synaptic release of dopamine within the striatum [25]. These mechanisms include synthesis and vesicular storage of dopamine by surviving nigrostriatal terminals, a decrease in presynaptic dopamine transporter that will prolong synaptic availability of dopamine and at later stages recruitment of nondopaminergic mechanisms, including serotonergic terminals and nonaminergic neurons, for conversion of levodopa to dopamine [26-28]. As PD advances and dopaminergic denervation increases, as a result of progressive nigrostriatal dopamine cell degeneration, the balance between these compensatory mechanisms shifts in a way that leads to erratic and nonphysiological release of extracellular dopamine. For example, as remaining dopaminergic terminals degenerate, nondopaminergic terminals play a more prominent role in conversion of exogenous levodopa to dopamine [27]. However, these terminals lack the feedback autoregulatory and reuptake mechanisms that control the physiological phasic release of dopamine into the synapse, thereby subjecting the synapse to unpredictable, unconstrained and protracted dopamine exposure. At this point, the relationship between fluctuating plasma levels of levodopa and central release of dopamine becomes more linear, leading to large swings in the amount of synaptic dopamine released during the first hour after each dose of levodopa, which correlate with the severity of peak-dose dyskinesia [29,30]. This pattern of swinging dopamine release in response to levodopa was present in subjects who subsequently developed dyskinesia as opposed to those who did not develop motor complications within 3 years [31]. The contribution of serotoninergic terminals in this process remains to be further clarified. It is known that in a dopamine-denervated state, the serotoninergic terminals can store dopamine and release it in a nonphysiological pattern [32]. However, studies using post-mortem serotonin markers have failed to show a difference in striatal serotonin levels between patients with and without dyskinesia [33].

The pulsatile and nonphysiological dopaminergic stimulation in turn sets into motion a number of downstream changes within the dopamine-denervated striatum that alter the output of the basal ganglia and lead to the development of dyskinesias. These downstream changes within the basal ganglia involve permanent modifications at multiple functional levels that range from long-term changes in synaptic connectivity within the striatum, to postsynaptic changes in signaling pathways, altered rate and pattern of neuronal firing and abnormal oscillatory activity within the basal ganglia network, leading to generation of abnormal motor programs [6]. At the synaptic level, the long-term exposure to levodopa impairs the plasticity of glutamatergic corticostriatal synapses by abolishing the process of long-term depression and impairing the synaptic capacity to reverse (depotentiate) previously induced long-term potentiation, making these synapses inflexible and unable to erase nonessential motor information $[34,35]$. These changes only occur in the setting of dopamine denervation and are dependent on the dose of levodopa. Notably, experimental rescue of long-term depression within these synapses, using phosphodiesterase inhibitors, such as zaprinast, can abolish levodopa-induced dyskinesia in parkinsonian rats [36]. These synaptic changes are then paralleled by alterations in intracellular signaling pathways affecting the phosphorylation cascade, including ERK1, DARPP32, and transcription factors, such as CREB and $\triangle F o s B$ [37,38]. These changes may serve as a form of molecular memory mediating altered patterns of striatal gene expression, including some that regulate glutamate transmission or are involved in transduction downstream from the dopamine receptors $[37,39,40]$. Other adaptations include structural remodeling of key striatal medium spiny neurons, causing a selective loss of dendritic spines and subcellular redistribution of glutamate receptors secondary to changes in scaffolding proteins [41-43]. All these processes contribute to impairment of sensorimotor integration by striatal GABAergic output neurons and subsequent alterations in the neuronal activity within basal ganglia circuitry.

Although simplified, the classic schematic model of basal ganglia function provides a 
helpful framework to better understand the changes that occur at the neuronal and network activity levels in terms of the induction of dyskinesia. This model consists of direct and indirect pathways that exert net facilitatory and inhibitory effects on motor cortical activity, respectively [44]. A main abnormality in PD involves loss of the inhibitory influence exerted by $\mathrm{D} 2$ dopamine receptors on the indirect striatal pathway. This results in a cascade of effects, including overactivation of the indirect striatal pathway, underactivation of the globus pallidus externa (GPe), overactivation of the subthalamic nucleus (STN), and overactivation of the globus pallidus interna (GPi), leading to a net inhibitory influence on thalamocortical motor activity. In addition, there is an underactivity of the D1-mediated direct pathway, further diminishing the net permissive influence of this pathway on motor circuits. The end result is an increase in the firing rate of GPi neurons beyond what is observed in intact nonparkinsonian subjects and at frequencies exceeding $80 \mathrm{~Hz}$ [45-47]. Dopaminergic medications restore the $\mathrm{ON}$ state by decreasing the firing rate of GPi neurons, but there seems to be an overcorrection of this process during dyskinetic episodes leading to GPi firing rates that are lower than those seen in normal controls $[48,49]$. In addition to the rate of neuronal firing, there are important changes in the pattern of neuronal activity. For example, in patients with PD with dyskinesia during ON state, the firing pattern of GPi neurons becomes irregular and bursty [48,50]. In addition to changes in the activity of individual neurons, PD is also associated with important alterations in the synchronized oscillatory activity within corticostriatal circuits [51,52]. These large-scale oscillations are markers of coordinated postsynaptic potentials between large ensembles of neurons, and exert a frequency-specific influence over the output of motor control systems. Patients with PD exhibit an excessive increase in the oscillatory activity within basal ganglia in the beta band range $(10-35 \mathrm{~Hz})[51]$. They also lack the phasic attenuation of beta synchrony between basal ganglia and motor cortex that characterizes voluntary movements in normal subjects, indicating a deficit in decoupling between the two regions that has been linked to slowness and rigidity in these patients $[52,53]$. Levodopa suppresses the abnormal oscillatory activity at the beta range and may facilitate prokinetic higher frequency oscillations at the gamma range [52]. In addition, it has been reported that periods of levodopa-induced dyskinesia are associated with an increase in the slower oscillatory activity within the $4-10 \mathrm{~Hz}$ frequency band in the contralateral STN [54]. These neurophysiological mechanisms serve as a functional end point in the process of expression of dyskinesia and targeting them by surgical interventions, such as deep brain stimulation (DBS) or pallidotomy, has proved effective in suppressing dyskinesia [55]. Moreover, drugs that can change the firing pattern and synchronization within these regions have the potential to reduce the expression of established dyskinesia.

Pharmacological research into differential mechanisms involving direct and indirect striatal pathways has further clarified a number of key neurotransmitter/receptor complexes that play distinct roles in the development versus expression of levodopa-induced dyskinesia. Stimulation of the D1-containing direct pathway is critical for development of levodopainduced dyskinesia and this pathway remains overactive during expression of established dyskinesia $[23,56]$. Structural studies have shown that in parkinsonian subjects with levodopa-induced dyskinesia, dopamine D1 receptors are highly expressed at the plasma membrane. This process may be a consequence of impaired receptor desensitization and internalization, and can mediate the observed behavioral supersensitivity to levodopa [56,57]. In addition, the D3 dopamine receptors, which are coexpressed with D1 receptors on the direct pathway, are also expressed at increased levels in subjects with dyskinesia, and their expression is linearly correlated with the severity of dyskinesia [58]. Pure D2 dopamine agonists, which target the indirect pathway, less commonly cause dyskinesia, but if dyskinesia is already established then D2 receptor stimulation can cause or exacerbate the expression of dyskinesia [12]. This finding may explain the clinical observation that early therapy with D2 dopamine receptor agonists, like pramipexole or ropinirole, rather than levodopa can delay the initiation of levodopa therapy and the subsequent development of dyskinesia, but once dyskinesia develops, agonists will exacerbate dyskinesia $[12,17]$. Furthermore, targeting putative communication mechanisms between direct and indirect pathways, including gap junctions and neuropeptide signaling through recurrent striatal collaterals, may contribute to prevention of dyskinesia development [6].

Other nondopaminergic systems, most notably glutamatergic pathways, are also implicated in the pathophysiology of dyskinesia. 
Corticostriatal transmission through both NMDA and AMPA glutamate receptors undergo changes in subjects with established dyskinesia [59-61]. Specifically, the level of NMDA receptors containing the NR2B subunit is increased in the setting of expression of dopamine drug-related dyskinesias in PD patients [62]. As mentioned above, dyskinesia is also associated with abnormal phosphorylation and altered synaptic trafficking of glutamate receptors [39]. Blockade of NMDA receptors is considered the main mode of action for the suppressing effect of amantadine on dyskinesia. Changes in the expression of opioid peptides is one of the most consistent findings in levodopainduced dyskinesia, although the mechanisms involved are complicated and receptor subtype specific. There is an increase in the level of the opioid peptide dynorphin in direct pathway neurons in association with the expression of dyskinesia, although it remains to be clarified if this is a causative or compensatory effect [6]. On the other hand, in the indirect pathway, the increased expression of another opioid peptide, preproenkephalin- $\mathrm{B}$, may have a facilitatory effect on the dyskinesia priming process. For example, in parkinsonian monkeys, initiating levodopa therapy during the window of elevation of preproenkephalin-B level leads to development of dyskinesia, whereas later onset of treatment outside this window does not lead to dyskinesia $[39,63]$. Interestingly, a recent study in MPTP-treated primates showed that a selective mu-opioid receptor antagonist suppressed dyskinesia, whereas previous studies with the nonselective opioid receptor antagonist, naloxone, had failed, further supporting the subtype-specific role of opioid receptors in dyskinesia [64]. Other changes in the direct pathway during dyskinesia involve alterations in $\alpha_{2}$-adrenergic, $5 \mathrm{HT}_{1 \mathrm{~A}}$ and $5 \mathrm{HT}_{2 \mathrm{~A}}$ serotonin, $\mathrm{H} 3$ histamine, and $\mathrm{CB} 1$ cannabinoid receptor transmission [39]. In addition, BDNF, a regulator of synaptic plasticity, may play a role in susceptibility to dyskinesia. Patients with the met allele of BDNF, which is associated with lower activity-dependent secretion of this molecule, are at significantly higher risk of developing dyskinesias earlier in the course of dopaminergic therapy [65]. Mechanisms for this effect of BDNF polymorphism on dyskinesia are not well understood but it has been postulated that lower BDNF activity leads to aberrant synaptic plasticity, possibly contributing to a decreased ability to potentiate and depotentiate corticostriatal synapses in met allele carriers [65].
Different mechanisms of dyskinesias

Given differences in the clinical manifestations of dyskinesias, it is likely that distinct mechanisms underlie the different types of dyskinesia. For example, in peak-dose dyskinesia, the key abnormality is felt to be overactivation of the direct pathway that leads to underactivity of basal ganglia output. However, distinct activation patterns involving GPe and STN lead to chorea or dystonia. In peak-dose chorea, excitatory subthalamic projections to GPi are underactivated, whereas in peak-dose dystonia these pathways demonstrate aberrant foci of overactivity. In diphasic dyskinesia, a concomitant overactivation of the indirect pathway along with the direct pathway has been postulated. In OFF-state dystonia, the overactivation of the indirect pathway plays a prominent role while the direct pathway is underactivated. Physiological changes underpinning this type of dyskinesia are similar to those in primary dystonia, including increased irregular firing in GPi $[66,67]$. The idea of distinct pathways underlying different types of dyskinesias is also supported by pharmacological evidence. Levodopa as well as dopamine receptor agonists can increase the duration of peak-dose dyskinesia and increase the first phase of diphasic dyskinesia but will improve the second phase of diphasic dyskinesia as well as OFF-state dystonia. The cannabinoid CB1 receptor agonists can decrease diphasic and possibly OFF-state dyskinesias but do not improve peak-dose dyskinesia, whereas the cannabinoid CB1 receptor antagonist rimonabant can suppress the expression of peak-dose dyskinesia [68]. These apparently paradoxical effects have been attributed to the primary effect of the cannabinoid agonist on the indirect pathway, correcting a maladaptive decrease in endocannabinoid levels in GPe, versus the predominant impact of the antagonist on the direct pathway, blocking the overstimulation of endocannabinoid receptors that accompany peak-dose dyskinesia [39]. Another example of differing mechanisms comes from the observation that histamine $\mathrm{H} 3$ receptor agonists can reduce choreic but not dystonic form of dyskinesia. Given multiple phenomenologies of dyskinesias, different therapeutic approaches may be required based on the predominant type of phenomenology.

\section{Treatment issues \\ Current medical therapies}

Available medical therapies for dyskinesia can be divided into those that treat the expression of levodopa-induced dyskinesia and those that can prevent it. The first step in the treatment of 
existing dyskinesia is an assessment of the clinical phenomenology of dyskinesias and their temporal relationship to levodopa dosing. The next step involves modification of PD medications. In peak-dose dyskinesia, a useful strategy involves decreasing the individual levodopa doses, possibly compensated by increasing the frequency of doses. If a patient is on a sustained release formulation of levodopa, switching to a standard short-acting formulation may help to regulate peak-dose dyskinesia by allowing the patient and clinician to document dyskinesia development or exacerbation relative to specific doses. Removing adjunct PD medications that act through prolonging the levodopa effect, including inhibitors of MAO-B and COMT, can also reduce peakdose dyskinesia. Sometimes a reduction in the dose of dopamine D2 receptor agonists may also be necessary. Addition of an antidyskinesia agent may also provide additional benefit. To date, only two agents, amantadine and clozapine have shown evidence-based efficacy against medication-induced dyskinesia [69,70]. Amantadine, as noted above, is a NMDA receptor blocker that can exert antidyskinetic effects without diminishing the antiparkinsonian effect of levodopa. Clozapine is an atypical antipsychotic drug with a complex mode of action that includes antagonism at D1, D2, D4 dopamine and 5 $\mathrm{HT}_{2}$ serotonin receptors and agonism at $5 \mathrm{HT}_{1 \mathrm{~A}}$ receptors. Given the risk of major adverse effects, notably agranulocytosis, currently the Movement Disorder Society Evidence-Based Medicine Reviews (MDS EBMR) practice implication for clozapine is possibly useful [69]. In advanced PD with severe peak-dose dyskinesia, additional helpful strategies include enteral infusion of a gel form of levodopa through an intraduodenal catheter [71], judged as 'likely efficacious' in a recent evidence-based review of treatments [69]. Continuous subcutaneous delivery of apomorphine has been reported to abate dyskinesias in some studies, although the clinical efficacy of this method remains to be fully established in controlled studies $[72,73]$. This method has been approved for refractory motor fluctuations in some European countries. The intestinal delivery of the gel formulation of levodopa (Duodopa ${ }^{\circledR}$ ), infused by a portable pump and delivered via a permanent percutaneous endoscopic gastrostomy tube inserted into the duodenum, offers an additional method for treatment of refractory dyskinesia and motor fluctuations [74]. This strategy has been approved for treatment of advanced PD with refractory dyskinesia and motor fluctuations in some European countries but not in the USA.
In OFF-state dyskinesia, usually dystonia, the main strategy is to reduce the OFF state itself. This strategy can be achieved by increasing the dosing or frequency of levodopa, adjunctive therapy with MAO-B or COMT inhibitors, adding D2 dopamine receptor agonists, and in refractory cases, subcutaneous apomorphine injections for rapid rescue from the OFF state. Sustained release levodopa can be helpful to relieve nocturnal painful OFF-state dystonia. Diphasic dyskinesia is more difficult to treat, but there are strategies available that can be tried, including using higher doses of short-acting levodopa, increasing adjunctive MAO-B or COMT inhibitors, and use of a very frequent dosing schedule at times with a liquid form of levodopa [6,75].

Available medical therapies for the prevention of dyskinesia are based on the concept that continuous dopaminergic stimulation may delay the development of dyskinesia through diminishing the intermittent and erratic dopaminergic stimulation [73,76]. Accordingly, dopamine D2 agonists, including their prolonged release formulations, have proved efficacious in delaying/preventing dyskinesia and should be considered as a useful clinical strategy. In a recent evidence-based medical review pramipexole, ropinirole (both short-acting and long-acting formulations) and cabergoline were designated as 'efficacious' in preventing dyskinesia [69]. Bromocriptine and pergolide were designated as 'likely efficacious'. So far, available controlled release formulations of levodopa, or adjunct use of the COMT inhibitor, entacapone, as well as the MAO-B inhibitor, selegiline, have proved 'nonefficacious' in preventing dyskinesia [69]. In fact, initiating levodopa therapy simultaneously with a COMT inhibitor is shown to shorten the time-to-onset and increase the frequency of dyskinesia, possibly reflecting the higher levodopa dose equivalents in the presence of COMT inhibitors [77]. There is insufficient data regarding the effects of amantadine or clozapine on the prevention of dyskinesia. Even with the available strategies, however, a sizable group of patients are insufficiently helped, necessitating the development of new strategies that draw upon our current understanding of the pathophysiology of drug-related dyskinesias.

\section{Investigational, medical \& surgical therapies}

A number of strategies have been under investigation for treatment of dyskinesia. One strategy involves modulation of the D3 dopamine receptors that are coexpressed with D1 receptors on 
the direct pathway. Partial agonists of the D3 dopamine receptor can reduce dyskinesia in animal models, without attenuating the therapeutic effect of levodopa [57,78]. Another strategy targets glutamatergic transmission. Dextrometorphan, an agent with NMDA antagonist properties, has been reported to possess antidyskinetic effects [79]. Riluzole and memantine, two other agents with anti-NMDA receptor properties have also been investigated for their antidyskinetic effects. AMPA receptor blockers, such as talampanel, have also shown promise in controlling dyskinesia in primate models [80]. A major problem associated with agents targeting NMDA or AMPA receptors is the ubiquitous distribution of these receptors, raising the risk of major adverse effects. This issue has directed attention towards another class of receptors, the metabotropic glutamate receptors (mGluRs), given their more restricted distribution and slower mode of action. In this regard, group $5 \mathrm{mGluR}$ is of special interest as these receptors are highly colocalized with NMDA receptors within corticostriatal circuits and can potentiate their transmission [81]. Accordingly, negative modulators of group 5 mGluRs have recently shown promising antidyskinetic properties without changing the antiparkinsonian effects of dopaminergic therapy [82]. Another potential strategy is based on targeting specific serotonin receptors. In this regard, a $5 \mathrm{HT}_{1 \mathrm{~A}}$ receptor agonist, sarizotan, has not shown antidyskinetic efficacy in two large studies despite some positive results in earlier trials. On the other hand, quetiapine, a $5 \mathrm{HT}_{2 \mathrm{~A}}$ receptor antagonist, has been promising in small studies. Other medications with anecdotal effects against the expression of dyskinesia include the $\alpha_{2}$-adrenergic antagonist idazoxan, cannabinoid agents nabilone and rimonabant and propanolol $[6,39]$. While the antiepileptic agent zonisamide has been efficacious as a symptomatic adjunct to levodopa, there is insufficient data regarding its efficacy against dyskinesia [83].

Investigational strategies for prevention of dyskinesia include D3 dopamine receptor antagonists, such as S33084, which have shown potential in preclinical studies. Antagonists selective for NMDA receptors containing the NR2B subunit can block the development of dyskinesia in primate models, although clinical efficacy of this approach remains to be established [84]. Strategies aimed at providing antiparkinsonian effects without dyskinesia can also help to decrease the need for levodopa and delay the development of dyskinesias. In this regard, one promising approach is the blockade of adenosine A2a receptors on indirect pathway. This mechanism bypasses the direct pathway that plays the key role in the development of dyskinesia. This method showed promise in animal studies and is currently under further clinical investigation using adenosine A2a receptor antagonists, such as istradefylline [85]. Similarly, dopamine transporter inhibitors, such as brasofensine and tesofensine, are under study, both as monotherapy and adjunct therapy, for their potential antiparkinsonian effects related to prolongation of the synaptic availability of dopamine [6]. A related line of research is focused on strategies that may allow the reversal of dyskinesia priming process by disrupting the mechanisms that maintain a state of increased sensitivity to levodopa, and other dopaminergic medications, once dyskinesia is established. In this regard, levitracetam, an antiepileptic agent, has proved effective in reversing the dyskinesia-priming after its establishment in parkinsonian primates [86]. A recent exploratory crossover study showed that levetiracetam could be useful in improving dyskinesia [87]. While the mechanism of action of levitracetam on dyskinesia priming remains unclear, its ability to block synaptic vesicle glycoprotein 2 , a regulator of presynaptic neurotransmitter release, has been implicated as a likely mechanism. Another potential mechanism with demonstrated ability to reverse dyskinesia priming in preclinical models involves glial cell line-derived neurotrophic factor [88]. Clinical studies to assess this mechanism are under way.

A separate line of investigational medical therapies for PD involve a number of ongoing gene therapy programs that include AAV2-neurturin, AAV2-GAD and AAV-hAADC. In a small noncontrolled study, bilateral intrastriatal infusion of an adeno-associated viral vector containing the human aromatic L-amino acid decarboxylase gene $(A A V-h A A D C)$ led to a significant increase in 'ON' time without any increase in ON-state dyskinesia [89]. Also, intraputaminal delivery of the gene for trophic factor neurturin (AAV2-neurturin) did not show any superiority over the sham group at 12 months as assessed by UPDRS motor score although there was a significant increase in the amount of $\mathrm{ON}$ time without dyskinesia [90]. By contrast, in a double-blind, Phase II, randomized controlled trial of intra-STN gene therapy with glutamic acid decarboxylase (AAV2-GAD), no change was noted in the dyskinesia score [91]. While this line of therapies remain investigational at present, preliminary data indicate that certain forms of 
gene therapy may have the potential to provide beneficial effects against dyskinesia.

\section{Other surgical therapies: lesions, deep brain stimulation \& cell grafts}

In addition to medical therapies, there are now surgical interventions that have proven efficacious for the treatment of dyskinesia and these include unilateral pallidotomy, bilateral STN DBS and bilateral GPi DBS [69]. Unilateral pallidotomy is an efficacious and clinically useful method for reduction of contralateral dyskinesia $[92,93]$. The main targets have included posteroventral as well as anteromedial sections of GPi. Bilateral pallidotomy is associated with major adverse effects and should be avoided because of safety concerns, except for exceptional cases [94]. There is insufficient evidence regarding efficacy of other ablative surgeries, including unilateral thalamotomy and unilateral or bilateral subthalamotomy. With advances in DBS, the focus of surgical treatment has shifted to this potentially reversible procedure. Both bilateral GPi and STN DBS are efficacious and clinically useful in treatment of dyskinesia. Bilateral GPi DBS can lead to an overall improvement in dyskinesia severity by $41-87 \%$ and increase in ON time without dyskinesia [95]. It should be noted that the stimulating electrode consists of four contacts. The stimulation of the most ventral contact can effectively block the dyskinesia but may worsen the underlying akinesia and block the antiparkinsonian effect of levodopa, whereas stimulation of the most dorsal contact alleviates akinesia but can worsen dyskinesia [12]. In recent years, STN has become the most commonly used target for DBS in patients with PD with severe dyskinesia or other motor complications. Bilateral STN DBS decreases the severity of all clinical patterns of dyskinesia, reduces OFF time and increases duration of ON time without dyskinesia $[96,97]$. In addition, STN DBS allows the dose of levodopa and other PD medications to be reduced. This reduction can in turn decrease the severity of dyskinesia [98]. Currently, there is insufficient evidence regarding the efficacy of thalamic DBS. Benefits of DBS implantation should be weighed against its associated adverse effects related to the procedure (intracranial hemorrhage, infection and infarction), device complications (malfunction, lead fractures, lead displacement and ulceration) and stimulation side effects (paresthesia, dysarthria, diplopia, muscle pulling, weight gain, and psychiatric and cognitive symptoms). Whereas surgical interventions have focused on treating pre-existing dyskinesia, no treatment has been studied in terms of preventing the eventual development of dyskinesia. At the current time, patients with short-term PD are not treated regularly with surgical intervention, so this type of information is not possible to acquire. As the use of DBS advances to include these milder patients, information on the prevention of dyskinesia with surgical treatments may be feasibly acquired with appropriate control or comparison groups.

Another line of experimental treatment for PD has included implantation of mesencephalic allografts within the striatum with the goal of providing a sustained source of dopamine. Some patients treated with this method have developed severe refractory dyskinesias, termed 'run-away dyskinesias', that occur even when patients do not take their regular dopaminergic medications [99]. A number of factors including severity of baseline dopaminergic denervation, location and tissue distribution of grafted cells, erratic nonphysiological dopamine release from the graft, excessive serotoninergic innervation of the graft, and possibly degree of immunosuppressants have been considered to influence graft-induced dyskinesia [99-101]. Importantly, functional imaging studies have indicated that, contrary to initial assumptions, this form of dyskinesia is not mediated by abnormal dopamine release by the graft $[101,102]$. Instead, a recent report indicated an increase in striatal serotonin to dopamine transporter ratio, and reversal of dyskinesia by a serotonin $5-\mathrm{HT}_{1 \mathrm{~A}}$ agonist in a patient with prolonged graft-induced dyskinesia [103]. Some refractory cases of graft-induced dyskinesia have been treated with DBS. Intraoperative recording during these DBS implantation surgeries has indicated that similar to levodopa-induced dyskinesia, graft-induced dyskinesia is associated with overactivity of neurons in the direct striatal pathway [104].

\section{Dyskinesia rating scales}

One of the main challenges facing the development of new treatments for dyskinesia is to ensure the objective measurement of dyskinesia in a reliable and consistent way. This effort has been complicated by the protean nature of dyskinesias, their temporal dynamics, and their interpersonal as well as intrapersonal variations. To address these concerns, several dyskinesia rating scales have been developed. These include the Abnormal Involuntary Movement Scale (AIMS), the Rush Dyskinesia Rating Scale (RDRS), UPDRS-IV, the Lang-Fahn Scale of patient perceptions, the Obeso scale, the PD-Dyskinesia 
26 questionnaire, and the Unified Dyskinesia Rating Scale (UDysRS). Some of these scales including AIMS, LFS, PD-dyskinesia 26 questionnaire and diaries rely on patients' perceptions while other scales such as RDRS, UPDRS and MDS-UPDRS assess the phenomenology of dyskinesia. RDRS and MDS-UPDRS also include rater's evaluation of dyskinesia-induced disability. However, none of these scales were sufficiently comprehensive to cover all pertinent aspects of dyskinesia rating [105]. In an effort to develop a comprehensive tool for assessment of dyskinesia, UDysRS was recently developed [106]. This scale includes all pertinent aspects related to the evaluation of dyskinesia including patient perceptions, impairment, disability, anatomic distribution and phenomenology and has been clinimetrically tested in a variety of programs [105].

UDysRS consists of 26 ratings that allow a total score between 0 and 140. Part I evaluates $\mathrm{ON}$ dyskinesia and consists of ten questionnaire items and one item for rating time spent with dyskinesia. Part II evaluates OFF-state dyskinesia and consists of three questionnaire items and one item for rating time spent with dyskinesia. Part III is an objective measure of the severity of dyskinesia and assesses the severity of dyskinesia in seven body regions on four tasks of daily living. Part IV consists of a disability rating on each of the four tasks. The clinimetric description of UDysRS was published in 2008 and indicated strong internal consistency, factor structure, inter-rater and intrarater reliability for each of the four parts of this scale [106]. Strengths of UDysRS include combining modified versions of established dyskinesia scales, covering both subjective and objective components and integration with MDS-UPDRS. Further clinimetric studies are needed to assess concurrent validity (UDysRS vs other scales), content validity (confirmatory factor analysis), discriminative validity (dyskinesia versus other movements), and treatment responsivity of UDysRS scale [107].

\section{Placebo effect}

Another potential problem complicating assessment of dyskinesia in controlled clinical trials is the potential impact of the placebo effect on dyskinesia. This is particularly relevant given the key role of the dopaminergic system in both processes. The dopaminergic system is involved in motivation, reward anticipation and response to novelty and change [108]. It is notable that the experience of participation in a clinical trial is anchored in the prospect of the use of a new medication or surgery and is associated with enhanced attention, anticipation of change, self encouragement and enhanced motivation. In addition, trials include increased numbers of clinical visits and regular clinical and laboratory assessments. All these factors can modify dopaminergic transmission, leading to striatal dopaminergic augmentation and improvement of parkinsonism [109]. It has been shown that the occupancy of postsynaptic dopamine D2 receptors by dopamine, as assessed by raclopride binding, is increased in placebo-treated responder patients but not in nonresponders, indicating the significance of dopamine pathways in mediating response to placebo [110]. If heightened dopaminergic activity occurs as part of placebo-associated improvements in parkinsonism, it is reasonable to predict that study participation would aggravate dyskinesia. In one study of a putative treatment for dyskinesia, however, placebo-associated improvements in dyskinesia occurred much more frequently than exacerbation, suggesting that a simple augmentation of dopamine is not the sole explanation for dyskinesia [109]. Clearly, multiple other neurotransmitter systems including glutamate (NMDA), GABA, serotonin, opioid, cannabinoid CB1 and histamine receptors have also been implicated as putative mediators of the placebo effect, and likewise, as discussed above, multiple neurochemicals modulate dyskinesia. As researchers pursue the chemical foundations of both placebo effects and dyskinesia, clinical trial designs must incorporate the reality of placebo-associated improvements into effect size estimates and sample size calculations for future dyskinesia studies.

\section{Future perspective}

Our emerging understanding of the complex pathophysiology underlying dyskinesia will guide future efforts directed at prevention and treatment of drug-induced dyskinesia. Multiple pharmacological targets, including several nondopaminergic neurotransmitter pathways, have been identified as potential strategies to treat dyskinesia after its establishment. These include glutamate-based mechanisms, such as negative allosteric modulators of group 5 metabotropic receptors, cannabinoid modulators, opioids and serotoninergic agents. These efforts could lead to the development of more effective treatments for dyskinesia as well as the selective targeting of specific types of dyskinesias based on their distinct underlying pharmacology. Efforts aimed at 
the prevention of dyskinesia include improved methods to provide continuous dopaminergic stimulation as well as employment of new nondopaminergic mechanisms, such as adenosine receptor antagonism, to exert antiparkinsonian effects without dyskinesia. Future advances in surgical treatment of dyskinesia will be directed at the better understanding of the physiological basis of dyskinesias, such as aberrant oscillatory overactivity in basal ganglia, and the refinement of methods to detect and selectively modulate these abnormalities. Finally, the application of rating scales that are both comprehensive and include an assessment of the functional impact of dyskinesias, as well as consideration of the placebo effect on dyskinesia expression, will allow improved design of clinical trials of dyskinesia.

\section{Executive summary}

\section{Phenomenology of dyskinesia}

- Dyskinesias can consist of various abnormal movements, but typically choreic or dystonic movements predominate.

- Dyskinesias can present in three major patterns based on their temporal relationship to the dosing of levodopa: peak-dose dyskinesia, diphasic dyskinesia and end-of-dose or OFF-state dystonia.

\section{Epidemiology \& impact on quality of life}

- The main risk factors for development of dyskinesia are severity and duration of Parkinson's disease, levodopa or other dopaminergic drug exposure (levodopa having a higher risk for dyskinesia onset than dopamine agonists), overall daily dose and frequency of dosing of levodopa therapy, young age at Parkinson's disease onset and female gender.

- Whereas the risk of dyskinesia onset is reduced with dopamine agonists in comparison to levodopa therapy, once dyskinesias develop, dopamine agonists will increase dyskinesias.

- Dyskinesias, especially the painful forms and those that impair activities of daily life, diminish quality of life and increase healthcare costs.

\section{Pathophysiology \& pharmacology}

- Distinct, though at times overlapping, mechanisms mediate the development and expression of medication-related dyskinesias in Parkinson's disease.

- Intermittent and erratic synaptic release of dopamine into a dopamine-denervated striatum leads to synaptic and downstream intracellular plasticity that results in the emergence of dyskinesia.

- While stimulation of both D1 and D2 dopamine receptors by levodopa is implicated in the process of dyskinesia, the overactivation of the D1-containing direct striatal pathway is essential for the expression of dyskinesia.

- Other nondopaminergic neurotransmitter systems, including glutamate, opioid, $\alpha_{2}$-adrenergic, histamine, serotonin and adenosine receptors all participate in the regulation of dyskinesia, providing potential pharmacological targets.

- Levodopa-induced dyskinesia is associated with altered physiology of basal ganglia and changes in firing rates, pattern and oscillatory activity of basal ganglia output neurons that can be targeted through surgical interventions, such as globus pallidus interna or subthalamic nucleus deep brain stimulation.

\section{Treatment issues}

- Current management strategies target distinct phenomenologies of dyskinesias.

- The 2011 Movement Disorder Society Evidence-Based Medicine Reviews (MDS EBMR) review identifies the following medications as efficacious for the treatment of dyskinesias: amantadine and clozapine for treatment of dyskinesia, and dopamine D2 receptor agonists, pramipexole, ropinirole and cabergoline, for prevention of dyskinesia.

- The 2011 MDS EBMR review identifies the following surgical interventions as efficacious for the treatment of dyskinesias: unilateral pallidotomy, bilateral subthalamic nucleus deep brain stimulation and bilateral globus pallidus interna deep brain stimulation.

- Various dyskinesia rating scales capture distinct aspects of dyskinesia, including anatomic distribution and phenomenology, impairment, disability, phenomenology and patient perceptions. The Unified Dyskinesia Rating Scale was recently developed as a comprehensive tool for assessment of dyskinesia.

- Dyskinesia may be inherently susceptible to the dopamine-modulating effects of placebo and this effect needs to be monitored during placebo-controlled trials.

\section{References}

1. Pechevis M, Clarke CE, Vieregge P et al. Effects of dyskinesias in Parkinson's disease on quality of life and health-related costs: a prospective European study. Eur. J. Neurol. 12(12), 956-963 (2005).

2. Luquin MR, Scipioni O, Vaamonde J, Gershanik O, Obeso JA. Levodopa-induced dyskinesias in Parkinson's disease: clinical and pharmacological classification. Mov. Disord. 7(2), 117-124 (1992).

3. Fahn S. The spectrum of levodopa-induced dyskinesias. Ann. Neurol. 47 (4 Suppl. 1), S2-S9 (2000).

4. Lewitt PA. Conjugate eye deviations as dyskinesias induced by levodopa in
Parkinson's disease. Mov. Disord. 13(4), 731-734 (1998).

5. Weiner WJ, Goetz CG, Nausieda PA, Klawans HL. Respiratory dyskinesias: extrapyramidal dysfunction and dyspnea. Ann. Intern. Med. 88(3), 327-331 (1978).

6. Fabbrini G, Brotchie JM, Grandas F, Nomoto M, Goetz CG. Levodopa-induced 
dyskinesias. Mov. Disord. 22(10), 1379-1389 (2007).

7. Rice JE, Antic R, Thompson PD. Disordered respiration as a levodopa-induced dyskinesia in Parkinson's disease. Mov. Disord. 17(3), 524-527 (2002).

8. Ruzicka E, Zarubova K, Nutt JG, Bloem BR. 'Silly walks' in Parkinson's disease: unusual presentation of dopaminergic-induced dyskinesias. Mov. Disord. 26(9), 1782-1784 (2011).

9. Fernandez HH, Friedman JH. Punding on L-dopa. Mov. Disord. 14(5), 836-838 (1999).

10. Jankovic J. Motor fluctuations and dyskinesias in Parkinson's disease: clinical manifestations. Mov. Disord. 20 (Suppl. 11), S11-S16 (2005).

11. Marconi R, Lefebvre-Caparros D, Bonnet AM, Vidailhet M, Dubois B, Agid Y. Levodopa-induced dyskinesias in Parkinson's disease phenomenology and pathophysiology. Mov. Disord. 9(1), 2-12 (1994).

12. Rascol O, Fabre N, Brefel-Courbon C, Ory-Mange F, Perez-Lloret S. Dyskinesias. In: The Encyclopedia of Movement Disorders. Kompoliti K, Verhagen Metman L (Eds). Academic Press, CA, USA, 350-361 (2010).

13. Eriksen N, Stark AK, Pakkenberg B. Age and Parkinson's disease-related neuronal death in the substantia nigra pars compacta. J. Neural Transm. Suppl. (73), 203-213 (2009).

14. Stefanova N, Bucke P, Duerr S, Wenning GK. Multiple system atrophy: an update. Lancet Neurol. 8(12), 1172-1178 (2009).

15. Sharma JC, Bachmann CG, Linazasoro G. Classifying risk factors for dyskinesia in Parkinson's disease. Parkinsonism Relat. Disord. 16(8), 490-497 (2010).

16. Schrag A, Quinn N. Dyskinesias and motor fluctuations in Parkinson's disease. A community-based study. Brain 123(Pt 11), 2297-2305 (2000).

17. Fahn S, Oakes D, Shoulson I et al. Levodopa and the progression of Parkinson's disease. N. Engl. J. Med. 351(24), 2498-2508 (2004).

18. Hauser RA, Mcdermott MP, Messing S. Factors associated with the development of motor fluctuations and dyskinesias in Parkinson disease. Arch. Neurol. 63(12), 1756-1760 (2006).

19. Kostic VS, Marinkovic J, Svetel M, Stefanova E, Przedborski S. The effect of stage of Parkinson's disease at the onset of levodopa therapy on development of motor complications. Eur. J. Neurol. 9(1), 9-14 (2002).

20. Wickremaratchi MM, Ben-Shlomo Y, Morris HR. The effect of onset age on the clinical features of Parkinson's disease. Eur. J. Neurol. 16(4), 450-456 (2009).
21. Sharma JC, Ross IN, Rascol O, Brooks D. Relationship between weight, levodopa and dyskinesia: the significance of levodopa dose per kilogram body weight. Eur. J. Neurol. 15(5), 493-496 (2008).

22. Damiano AM, McGrath MM, Willian MK et al. Evaluation of a measurement strategy for Parkinson's disease: assessing patient health-related quality of life. Qual. Life Res. 9(1), 87-100 (2000).

23. Bezard E, Brotchie JM, Gross CE. Pathophysiology of levodopa-induced dyskinesia: potential for new therapies. Nat. Rev. Neurosci. 2(8), 577-588 (2001).

24. Schneider JS. Levodopa-induced dyskinesias in parkinsonian monkeys: relationship to extent of nigrostriatal damage. Pharmacol. Biochem. Behav. 34(1), 193-196 (1989).

25. Nutt JG, Holford NH. The response to levodopa in Parkinson's disease: imposing pharmacological law and order. Ann. Neurol. 39(5), 561-573 (1996).

26. Zigmond MJ, Abercrombie ED, Berger TW, Grace AA, Stricker EM. Compensations after lesions of central dopaminergic neurons: some clinical and basic implications. Trends Neurosci. 13(7), 290-296 (1990).

27. Carta M, Carlsson T, Kirik D, Bjorklund A. Dopamine released from 5-HT terminals is the cause of L-DOPA-induced dyskinesia in parkinsonian rats. Brain $130(\mathrm{Pt} 7)$, 1819-1833 (2007).

28. Brotchie J, Fitzer-Attas C. Mechanisms compensating for dopamine loss in early Parkinson disease. Neurology 72 (Suppl. 7), S32-S38 (2009).

29. De La Fuente-Fernandez R, Sossi V, Huang Z et al. Levodopa-induced changes in synaptic dopamine levels increase with progression of Parkinson's disease: implications for dyskinesias. Brain 127(Pt 12), 2747-2754 (2004).

30. Pavese N, Evans AH, Tai YF et al. Clinical correlates of levodopa-induced dopamine release in Parkinson disease: a PET study. Neurology 67(9), 1612-1617 (2006).

31. De La Fuente-Fernandez R, Lu JQ, Sossi V et al. Biochemical variations in the synaptic level of dopamine precede motor fluctuations in Parkinson's disease: PET evidence of increased dopamine turnover. Ann. Neurol. 49(3), 298-303 (2001).

32. Maeda T, Nagata K, Yoshida Y, Kannari K. Serotonergic hyperinnervation into the dopaminergic denervated striatum compensates for dopamine conversion from exogenously administered L-DOPA. Brain Res. 1046(1-2), 230-233 (2005).

33. Kish SJ, Tong J, Hornykiewicz $\mathrm{O}$ et al. Preferential loss of serotonin markers in caudate versus putamen in Parkinson's disease. Brain 131(Pt 1), 120-131 (2008).

34. Kreitzer AC, Malenka RC. Endocannabinoidmediated rescue of striatal LTD and motor deficits in Parkinson's disease models. Nature 445(7128), 643-647 (2007).

35. Picconi B, Paille V, Ghiglieri V et al. L-DOPA dosage is critically involved in dyskinesia via loss of synaptic depotentiation. Neurobiol. Dis. 29(2), 327-335 (2008).

36. Picconi B, Bagetta V, Ghiglieri V et al. Inhibition of phosphodiesterases rescues striatal long-term depression and reduces levodopa-induced dyskinesia. Brain 134(Pt 2), 375-387 (2011).

37. Jenner P. Molecular mechanisms of L-DOPA-induced dyskinesia. Nat. Rev. Neurosci. 9(9), 665-677 (2008).

38. Oh JD, Chase TN. Glutamate-mediated striatal dysregulation and the pathogenesis of motor response complications in Parkinson's disease. Amino Acids 23(1-3), 133-139 (2002).

39. Brotchie JM. Nondopaminergic mechanisms in levodopa-induced dyskinesia. Mov. Disord. 20 (8), 919-931 (2005).

40. Dunah AW, Wang Y, Yasuda RP et al. Alterations in subunit expression, composition, and phosphorylation of striatal $N$-methyl-D-aspartate glutamate receptors in a rat 6-hydroxydopamine model of Parkinson's disease. Mol. Pharmacol. 57(2), 342-352 (2000).

41. Gardoni F, Ghiglieri V, Luca M, Calabresi P. Assemblies of glutamate receptor subunits with post-synaptic density proteins and their alterations in Parkinson's disease. Prog. Brain Res. 183, 169-182 (2010).

42. Deutch AY. Striatal plasticity in parkinsonism: dystrophic changes in medium spiny neurons and progression in Parkinson's disease. J. Neural. Transm. Suppl. 70, 67-70 (2006).

43. Day M, Wang Z, Ding J et al. Selective elimination of glutamatergic synapses on striatopallidal neurons in Parkinson disease models. Nat. Neurosci. 9(2), 251-259 (2006).

44. Alexander GE, Delong MR, Strick PL. Parallel organization of functionally segregated circuits linking basal ganglia and cortex. Annu. Rev. Neurosci. 9, 357-381 (1986).

45. Magnin M, Morel A, Jeanmonod D. Single-unit analysis of the pallidum, thalamus and subthalamic nucleus in parkinsonian patients. Neuroscience 96(3), 549-564 (2000).

46. Starr PA, Rau GM, Davis V et al. Spontaneous pallidal neuronal activity in human dystonia: comparison with Parkinson's disease and 
normal macaque. J. Neurophysiol. 93(6), 3165-3176 (2005).

47. Sterio D, Beric A, Dogali M, Fazzini E, Alfaro G, Devinsky O. Neurophysiological properties of pallidal neurons in Parkinson's disease. Ann. Neurol. 35(5), 586-591 (1994).

48. Lee JI, Verhagen Metman L, Ohara S, Dougherty PM, Kim JH, Lenz FA. Internal pallidal neuronal activity during mild drug-related dyskinesias in Parkinson's disease: decreased firing rates and altered firing patterns. J. Neurophysiol. 97(4), 2627-2641 (2007).

49. Levy R, Dostrovsky JO, Lang AE, Sime E, Hutchison WD, Lozano AM. Effects of apomorphine on subthalamic nucleus and globus pallidus internus neurons in patients with Parkinson's disease. J. Neurophysiol. 86(1), 249-260 (2001).

50. Merello M, Balej J, Delfino M, Cammarota A, Betti O, Leiguarda R. Apomorphine induces changes in GPi spontaneous outflow in patients with Parkinson's disease. Mov. Disord. 14(1), 45-49 (1999).

51. Brown P. Oscillatory nature of human basal ganglia activity: relationship to the pathophysiology of Parkinson's disease. Mov. Disord. 18(4), 357-363 (2003).

52. Lalo E, Thobois S, Sharott A et al. Patterns of bidirectional communication between cortex and basal ganglia during movement in patients with Parkinson disease. J. Neurosci. 28(12), 3008-3016 (2008).

53. Wingeier B, Tcheng T, Koop MM, Hill BC, Heit G, Bronte-Stewart HM. Intra-operative STN DBS attenuates the prominent beta rhythm in the STN in Parkinson's disease. Exp. Neurol. 197(1), 244-251 (2006).

54. Alonso-Frech F, Zamarbide I, Alegre $\mathrm{M}$ et al. Slow oscillatory activity and levodopa-induced dyskinesias in Parkinson's disease. Brain 129(Pt 7), 1748-1757 (2006).

55. Bronte-Stewart H, Barberini C, Koop MM, Hill BC, Henderson JM, Wingeier B. The STN beta-band profile in Parkinson's disease is stationary and shows prolonged attenuation after deep brain stimulation. Exp. Neurol. 215(1), 20-28 (2009).

56. Guigoni C, Doudnikoff E, Li Q, Bloch B, Bezard E. Altered D(1) dopamine receptor trafficking in parkinsonian and dyskinetic non-human primates. Neurobiol. Dis. 26(2), 452-463 (2007).

57. Bezard E, Ferry S, Mach U et al. Attenuation of levodopa-induced dyskinesia by normalizing dopamine D3 receptor function. Nat. Med. 9(6), 762-767 (2003).

58. Berthet A, Bezard E. Dopamine receptors and L-dopa-induced dyskinesia. Parkinsonism Relat. Disord. 15(Suppl. 4), S8-S12 (2009).
59. Chase TN, Bibbiani F, Oh JD. Striatal glutamatergic mechanisms and extrapyramidal movement disorders. Neurotox. Res. 5(1-2), 139-146 (2003).

60. Konitsiotis S, Blanchet PJ, Verhagen L, Lamers E, Chase TN. AMPA receptor blockade improves levodopa-induced dyskinesia in MPTP monkeys. Neurology 54(8), 1589-1595 (2000).

61. Metman LV, Konitsiotis S, Chase TN. Pathophysiology of motor response complications in Parkinson's disease: hypotheses on the why, where, and what. Mov. Disord. 15(1), 3-8 (2000).

62. Calon F, Rajput AH, Hornykiewicz O, Bedard PJ, Di Paolo T. Levodopa-induced motor complications are associated with alterations of glutamate receptors in Parkinson's disease. Neurobiol. Dis. 14(3), 404-416 (2003).

63. Schneider JS, Gonczi H, Decamp E. Development of levodopa-induced dyskinesias in parkinsonian monkeys may depend upon rate of symptom onset and/or duration of symptoms. Brain Res. 990(1-2), 38-44 (2003).

64. Koprich JB, Fox SH, Johnston TH et al. The selective mu-opioid receptor antagonist ADL5510 reduces levodopa-induced dyskinesia without affecting antiparkinsonian action in MPTP-lesioned macaque model of Parkinson's disease. Mov. Disord. 26(7), 1225-1233 (2011).

65. Foltynie T, Cheeran B, Williams-Gray CH et al. BDNF Val66Met influences time to onset of levodopa induced dyskinesia in Parkinson's disease. J. Neurol. Neurosurg. Psychiatry 80(2), 141-144 (2009).

66. Hashimoto T, Tada T, Nakazato F et al. Abnormal activity in the globus pallidus in off-period dystonia. Ann. Neurol. 49(2), 242-245 (2001).

67. Sanghera MK, Grossman RG, Kalhorn CG, Hamilton WJ, Ondo WG, Jankovic J. Basal ganglia neuronal discharge in primary and secondary dystonia in patients undergoing pallidotomy. Neurosurgery 52(6), 1358-1370; discussion 1370-1373 (2003).

68. Van Der Stelt M, Fox SH, Hill M et al. A role for endocannabinoids in the generation of parkinsonism and levodopa-induced dyskinesia in MPTP-lesioned non-human primate models of Parkinson's disease. FASEB J. 19(9), 1140-1142 (2005).

69. Fox SH, Katzenschlager R, Lim S et al. Movement Disorder Society evidence-based medicine review update: treatments for the motor symptoms of Parkinson's disease. Mov. Disord. (Suppl. 26), S2-S41 (2011).

70. Durif F, Debilly B, Galitzky M et al. Clozapine improves dyskinesias in Parkinson disease: a double-blind, placebo-controlled study. Neurology 62(3), 381-388 (2004).

71. Nyholm D, Aquilonius SM. Levodopa infusion therapy in Parkinson disease: state of the art in 2004. Clin. Neuropharmacol. 27(5), 245-256 (2004).

72. Katzenschlager R, Hughes A, Evans A et al. Continuous subcutaneous apomorphine therapy improves dyskinesias in Parkinson's disease: a prospective study using single-dose challenges. Mov. Disord. 20(2), 151-157 (2005).

73. Olanow CW, Obeso JA, Stocchi F. Continuous dopamine-receptor treatment of Parkinson's disease: scientific rationale and clinical implications. Lancet Neurol. 5(8), 677-687 (2006).

74. Fernandez HH, Odin P. Levodopa-carbidopa intestinal gel for treatment of advanced Parkinson's disease. Curr. Med. Res. Opin. 27(5), 907-919 (2011).

75. Waters C. Other pharmacological treatments for motor complications and dyskinesias. Mov. Disord. 20(Suppl. 11), S38-S44 (2005).

76. Stocchi F. The therapeutic concept of continuous dopaminergic stimulation (CDS) in the treatment of Parkinson's disease. Parkinsonism Relat. Disord. 15(Suppl. 3), S68-S71 (2009).

77. Stocchi F, Rascol O, Kieburtz K et al. Initiating levodopa/carbidopa therapy with and without entacapone in early Parkinson disease: the STRIDE-PD study. Ann. Neurol. 68(1), 18-27 (2010).

78. Riddle LR, Kumar R, Griffin SA, Grundt P, Newman AH, Luedtke RR. Evaluation of the D3 dopamine receptor selective agonist/partial agonist PG01042 on L-dopa dependent animal involuntary movements in rats. Neuropharmacology 60 (2-3), 284-294 (2011).

79. Verhagen Metman L, Del Dotto P, Natte R, Van Den Munckhof P, Chase TN. Dextromethorphan improves levodopainduced dyskinesias in Parkinson's disease. Neurology 51(1), 203-206 (1998).

80. Konitsiotis S, Blanchet PJ, Verhagen L, Lamers E, Chase TN. AMPA receptor blockade improves levodopa-induced dyskinesia in MPTP monkeys. Neurology 54(8), 1589-1595 (2000).

81. Conn PJ, Battaglia G, Marino MJ, Nicoletti F. Metabotropic glutamate receptors in the basal ganglia motor circuit. Nat. Rev. Neurosci. 6(10), 787-798 (2005).

82. Berg D, Godau J, Trenkwalder C et al. AFQ056 treatment of levodopa-induced dyskinesias: results of 2 randomized controlled trials. Mov. Disord. 26(7), 1243-1250 (2011).

83. Murata M, Hasegawa K, Kanazawa I. Zonisamide improves motor function in 
Parkinson disease: a randomized, double-blind study. Neurology 68(1), 45-50 (2007).

84. Morissette M, Dridi M, Calon F et al. Prevention of levodopa-induced dyskinesias by a selective NR1A/2B $N$-methyl-D-aspartate receptor antagonist in parkinsonian monkeys: implication of preproenkephalin. Mov. Disord. 21(1), 9-17 (2006).

85. Mizuno Y, Hasegawa K, Kondo T, Kuno S, Yamamoto M. Clinical efficacy of istradefylline (KW-6002) in Parkinson's disease: a randomized, controlled study. Mov. Disord. 25(10), 1437-1443 (2010).

86. Hill MP, Brotchie JM, Crossman AR et al. Levetiracetam interferes with the L-dopa priming process in MPTP-lesioned drug-naive marmosets. Clin. Neuropharmacol. 27(4), 171-177 (2004).

87. Stathis P, Konitsiotis S, Tagaris G, Peterson D. Levetiracetam for the management of levodopa-induced dyskinesias in Parkinson's disease. Mov. Disord. 26(2), 264-270 (2011).

88. Rangasamy SB, Soderstrom K, Bakay RA, Kordower JH. Neurotrophic factor therapy for Parkinson's disease. Prog. Brain Res. 184, 237-264 (2010).

89. Christine CW, Starr PA, Larson PS et al. Safety and tolerability of putaminal AADC gene therapy for Parkinson disease. Neurology 73(20), 1662-1669 (2009).

90. Marks WJ Jr, Bartus RT, Siffert J et al. Gene delivery of AAV2-neurturin for Parkinson's disease: a double-blind, randomised, controlled trial. Lancet Neurol. 9(12), 1164-1172 (2010).

91. Lewitt PA, Rezai AR, Leehey MA et al. AAV2-GAD gene therapy for advanced Parkinson's disease: a double-blind, sham-surgery controlled, randomised trial. Lancet Neurol. 10 (4), 309-319 (2011).
92. Vitek JL, Bakay RA, Freeman A et al. Randomized trial of pallidotomy versus medical therapy for Parkinson's disease. Ann. Neurol. 53(5), 558-569 (2003).

93. Metman LV, O'leary ST. Role of surgery in the treatment of motor complications. Mov. Disord. 20(Suppl. 11), S45-S56 (2005).

94. Foltynie T, Hariz MI. Surgical management of Parkinson's disease. Expert Rev. Neurother. 10(6), 903-914 (2010).

95. Deep-Brain Stimulation for Parkinson Disease Study Group. Deep-brain stimulation of the subthalamic nucleus or the pars interna of the globus pallidus in Parkinson's disease. N. Engl. J. Med. 345(13), 956-963 (2001).

96. Kleiner-Fisman G, Herzog J, Fisman DN et al. Subthalamic nucleus deep brain stimulation: summary and meta-analysis of outcomes. Mov. Disord. 21(Suppl. 14), S290-S304 (2006).

97. Deuschl G, Herzog J, Kleiner-Fisman G et al. Deep brain stimulation: postoperative issues. Mov. Disord. 21(Suppl. 14), S219-S237 (2006).

98. Follett KA, Weaver FM, Stern M et al. Pallidal versus subthalamic deep-brain stimulation for Parkinson's disease. N. Engl. J. Med. 362(22), 2077-2091 (2010).

99. Lane EL, Bjorklund A, Dunnett SB, Winkler C. Neural grafting in Parkinson's disease unraveling the mechanisms underlying graft-induced dyskinesia. Prog. Brain Res. 184, 295-309 (2010).

100. Hudson JL, Hoffman A, Stromberg I, Hoffer BJ, Moorhead JW. Allogeneic grafts of fetal dopamine neurons: behavioral indices of immunological interactions. Neurosci. Lett. 171(1-2), 32-36 (1994).

101. Piccini P, Pavese N, Hagell P et al. Factors affecting the clinical outcome after neural transplantation in Parkinson's disease. Brain 128(Pt 12), 2977-2986 (2005).

102. Hagell P, Piccini P, Bjorklund A et al. Dyskinesias following neural transplantation in Parkinson's disease. Nat. Neurosci. 5(7), 627-628 (2002).

103. Politis M, Oertel WH, Wu K et al. Graftinduced dyskinesias in Parkinson's disease: high striatal serotonin/dopamine transporter ratio. Mov. Disord. 26(11), 1997-2003 (2011).

104. Richardson RM, Freed CR, Shimamoto SA, Starr PA. Pallidal neuronal discharge in Parkinson's disease following intraputamenal fetal mesencephalic allograft. J. Neurol. Neurosurg. Psychiatry 82(3), 266-271 (2011).

105. Colosimo C, Martinez-Martin P, Fabbrini G et al. Task force report on scales to assess dyskinesia in Parkinson's disease: critique and recommendations. Mov. Disord. 25(9), 1131-1142 (2010).

106. Goetz CG, Nutt JG, Stebbins GT. The unified dyskinesia rating scale: presentation and clinimetric profile. Mov. Disord. 23(16), 2398-2403 (2008).

107. Goetz CG, Stebbins GT, Theeuwes A et al. Temporal stability of the Unified Dyskinesia Rating Scale. Mov. Disord. 26(14), 2556-2559 (2011).

108. Schultz W. Multiple dopamine functions at different time courses. Annu. Rev. Neurosci. 30, 259-288 (2007).

109. Goetz CG, Wuu J, Mcdermott MP et al. Placebo response in Parkinson's disease: comparisons among 11 trials covering medical and surgical interventions. Mov. Disord. 23(5), 690-699 (2008).

110. De La Fuente-Fernandez R, Schulzer M, Stoessl AJ. The placebo effect in neurological disorders. Lancet Neurol. 1(2), 85-91 (2002). 


\section{Medscape Facing the unique challenges of dyskinesias in Parkinson's disease}

To obtain credit, you should first read the journal article. After reading the article, you should be able to answer the following, related, multiple-choice questions. To complete the questions (with a minimum $70 \%$ passing score) and earn continuing medical education (CME) credit, please go to www.medscape.org/journal/fnl. Credit cannot be obtained for tests completed on paper, although you may use the worksheet below to keep a record of your answers. You must be a registered user on Medscape.org. If you are not registered on Medscape.org, please click on the New Users: Free Registration link on the left hand side of the website to register. Only one answer is correct for each question. Once you successfully answer all post-test questions you will be able to view and/or print your certificate. For questions regarding the content of this activity, contact the accredited provider,
CME@medscape.net. For technical assistance, contactCME@webmd.net. American Medical Association's Physician's Recognition Award (AMA PRA) credits are accepted in the US as evidence of participation in CME activities. For further information on this award, please refer to http://www.ama-assn.org/ ama/pub/category/2922.html. The AMA has determined that physicians not licensed in the US who participate in this CME activity are eligible for AMA PRA Category 1 Credits ${ }^{\mathrm{TM}}$. Through agreements that the AMA has made with agencies in some countries, AMA PRA credit may be acceptable as evidence of participation in CME activities. If you are not licensed in the US, please complete the questions online, print the AMA PRA CME credit certificate and present it to your national medical association for review.

\section{Activity evaluation: where 1 is strongly disagree and 5 is strongly agree.}

The activity supported the learning objectives.

The material was organized clearly for learning to occur.

The content learned from this activity will impact my practice.

The activity was presented objectively and free of commercial bias.

1. Your patient is a 58-year-old woman with advanced Parkinson disease who is now being evaluated for involuntary movements thought to be dyskinesias. On the basis of the review by Drs. Homayoun and Goetz, which of the following statements about clinical features of dyskinesias is most likely correct?
A Myoclonus is the most common presentation of dyskinesia
B Choreic movements refer to distorted posturing
C Dystonic movements typically involve fast, irregular, jerking movements of the face, neck, and extremities
D Dyskinesias can occur as peak dose dyskinesia, diphasic dyskinesia, and end-of-dose or OFF-state dystonia

2. On the basis of the review by Drs. Homayoun and Goetz, which of the following statements about risk factors for dyskinesias in Parkinson disease is most likely correct?
A Severity and duration of Parkinson disease and exposure to levodopa or other dopaminergic drugs affect risk for dyskinesias
B The risk for dyskinesia onset is increased with dopamine agonists vs levodopa therapy
C Older age at onset of Parkinson disease increases risk for dyskinesias
D Male sex increases risk for dyskinesias 
3. On the basis of the review by Drs. Homayoun and Goetz, which of the following statements would most likely apply to management of the patient described in question 1?
A Pramipexole and ropinirole are effective to treat dyskinesias
B Amantadine and clozapine are not effective to treat dyskinesias
C Unilateral pallidotomy, bilateral subthalamic nucleus deep brain stimulation (DBS), or bilateral globus pallidus interna DBS may be effective to treat dyskinesias
D Placebo effect is negligible in patients with dyskinesias 